\title{
Une approche théorique de l'événementiel sportif
}

\author{
Olivier Bessy et André Suchet
}

\section{OpenEdition}

\section{Journals}

Édition électronique

URL : https://journals.openedition.org/tourisme/1023

DOI : $10.4000 /$ tourisme 1023

ISSN : 2492-7503

\section{Éditeur}

Association Mondes du tourisme

Référence électronique

Olivier Bessy et André Suchet, « Une approche théorique de l'événementiel sportif », Mondes du Tourisme [En ligne], 11 | 2015, mis en ligne le 01 décembre 2015, consulté le 21 septembre 2021. URL http://journals.openedition.org/tourisme/1023 ; DOI : https://doi.org/10.4000/tourisme.1023

Ce document a été généré automatiquement le 21 septembre 2021.

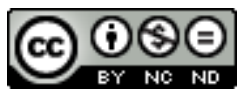

Mondes du tourisme est mis à disposition selon les termes de la licence Creative Commons Attribution - Pas d'Utilisation Commerciale - Pas de Modification 4.0 International. 


\title{
Une approche théorique de l'événementiel sportif
}

\author{
Olivier Bessy et André Suchet
}

1 Tour de France, X Games, Ultra-Trail du Mont-Blanc, Jeux olympiques, Internationaux de Roland-Garros, Natural Games, Lacanau Pro... le lancement d'une compétition sportive internationale est assurément un moyen de développement des territoires, mais encore faut-il s'entendre sur le type de développement auquel on veut parvenir et sur les moyens dont on se dote pour ce faire. Effectivement, si les liens entre sport, tourisme et développement à travers l'événement ont fait l'objet de très nombreuses analyses en sciences de gestion ou en économie - notamment les fameuses études sur les retombées d'un événement qui constituent quasiment un genre à part entière -, il reste des aspects à mieux théoriser au plan social et culturel.

2 Plus précisément, nombre d'études en sociologie ou en géographie sociale relatives au sport et au tourisme mobilisent un cadre théorique existant afin de comprendre l'événementiel sportif à travers ce prisme, et surtout afin de continuer à tester ce dispositif théorique. On peut citer Lacroix (1998) et son approche sensible du lien social au sein du Raid Passion Hérault, Kay et Laberge (2002) avec un travail sur l'habitus des participants au Discovery Channel Eco-Challenge, ou encore Obin et Corneloup (2014) qui mobilisent la théorie de l'acteur-réseau pour étudier la mise en place des Natural Games de Millau. L'ouvrage dirigé par Di Méo (2001) sous le titre La Géographie en fêtes est aussi un exemple de cette démarche; chacun des textes de ce volume collectif qui traite des événements festifs populaires ou traditionnels en France a pour intention d'alimenter les concepts forgés par Di Méo, et notamment celui de "formation socio-spatiale".

3 Ce n'est pas le sens de ce travail qui propose de partir des données accumulées au cours de différentes études de cas pour formaliser un dispositif théorique en trois axes afin de rendre compte de manière inédite de certains rapports entre un événement temporaire, des individus et un espace plus ou moins territorialisé. Après avoir pointé la difficulté à définir et à sous-catégoriser le monde de l'événementiel sportif, il s'agit donc d'axiomatiser : les trois niveaux d'effet d'un événement (premier axe); les deux grandes stratégies d'orientation possibles au départ du projet (second axe); et l'opposition 
structurelle entre un événement importé et un événement fabriqué (troisième et dernier axe).

4 La présente recherche, délibérément inductive, repose sur différentes études de cas menées sur la période 1990-2014 par les deux auteurs de ce texte - en particulier, le Marathon du Médoc depuis les années 1990 (Bessy, 1995, 2000), les Jeux pyrénéens de l'aventure 1993 (Suchet, 2012 ; Suchet, Jorand et Tuppen, 2010), le Grand Raid de la Réunion (Bessy, 2002, 2005a) et l'Ultra-Trail du Mont-Blanc (Bessy, 2012, 2014c). Il s'agit d'événements portés en France, et organisés sur le territoire national (métropole et outre-mer) ou en situation transfrontalière avec l'Italie, la Suisse et l'Espagne.

5 Le Marathon des châteaux du Médoc et des Graves, couramment nommé "Marathon du Médoc" est fondé en 1985 par des médecins et des négociants en vin qui "s'ennuyaient dans les marathons classiques" (Bessy, 1994, 2000). Le Marathon du Médoc est donc pensé comme une épreuve festive qui s'apparente au fil des ans à un véritable "carnaval de la course à pied" (déguisements, dégustations de vins et de produits régionaux, mais aussi animations musicales et folkloriques, avant, pendant et après la course). Dans ce premier cas d'étude, le recueil des données s'appuie sur une analyse de contenu des archives du marathon, de la première édition en 1985 à la quinzième en 1999, sur une vingtaine d'entretiens semidirectifs auprès des différents acteurs de cette manifestation, ainsi que sur une approche ethnographique et non directive longue, résultant de la participation à la course, à dix reprises, par un des auteurs de ce texte.

6 Les Jeux pyrénéens de l'aventure, ou los Juegos pirenaicos de la aventura, sont l'une des grandes compétitions sportives de nature, d'aventure et de freestyle organisées au début des années 1990. Cette compétition internationale qui s'est déroulée en 1993 sur la frontière entre la France et l'Espagne, soutenue par l'ensemble du mouvement olympique en Europe et patronnée par le CIO, se voulait la figure de proue d'un projet de développement transfrontalier, sportif et touristique des Pyrénées, tout en contribuant à rajeunir le mouvement olympique (Suchet, 2012 ; Suchet et Jorand, 2014). Un projet resté sans lendemain. Pour ce cas d'étude, nous avons procédé par analyse systématique des archives de l'organisation, avec une vingtaine d'entretiens semi-directifs ainsi qu'une observation sur le terrain et une étude des restes matériels et mémoriels de cette manifestation passée.

7 Le Grand Raid de La Réunion, ou "Diagonale des fous", est un raid pédestre balisé d'environ $160 \mathrm{~km}$ de long et $8000 \mathrm{~m}$ de dénivelé positif, qui traverse l'île du sud au nord en une seule étape (Bessy et Naria, 2004 ; Bessy, 2005a). Depuis quelques années, deux autres courses s'ajoutent au projet : le Semi-Raid de La Réunion, devenu Trail de Bourbon (100 km et $5000 \mathrm{~m}$ de dénivelé positif), et La Mascareignes ( $60 \mathrm{~km}$ et $2500 \mathrm{~m}$ ). Nous avons multiplié les investigations sur cet événement : étude des archives de la première édition en 1989 à la quatorzième en 2002, entretiens semi-directifs (au nombre d'une vingtaine) et phases d'observation participante ou non participante.

8 Quatrième et dernier cas d'étude, The North Face, Ultra-Trail du Mont-Blanc (Bessy, 2012). Cet événement comprend cinq épreuves de course-marche qui se déroulent autour du Mont-Blanc en traversant trois pays, la France l'Italie et la Suisse : l'Ultra-trail du Mont-Blanc ou UTMB, qui représente l'épreuve mythique créée en 2003 ; la CCC, ou Courmayeur-Champex-Chamonix, surnommée "la petite" ( $98 \mathrm{~km}$ et $5600 \mathrm{~m}$ de dénivelé positif); la TDS (sur les traces des ducs de Savoie), qui se déroule de Courmayeur à Chamonix (110 km et $7200 \mathrm{~m}$ de dénivelé positif) ; la PTL (la petite trotte à Léon), $290 \mathrm{~km}$ par équipes de deux ou trois coureurs sans classement final (18 $000 \mathrm{~m}$ de dénivelé positif), 
et, depuis cette année, l'OCC (Orsières-Champex-Chamonix), qui débute en Suisse autour du village d'Orsières (53 km et $3300 \mathrm{~m}$ de dénivelé positif). Les données de ce dernier cas d'étude proviennent de cinquante entretiens semi-directifs, réalisés auprès des créateurs et organisateurs de l'événement ainsi qu'auprès des principaux acteurs impliqués dans l'Espace Mont-Blanc. Données complétées par une analyse de contenu des plaquettes de présentation de la course et des articles de presse publiés à son sujet ainsi que par différentes phases d'observation participante.

Enfin, une dizaine d'autres manifestations ont bénéficié de notre attention au cours de ces recherches, notamment Réunion d'Aventures (éditions 2007 et 2009), le Championnat ou Course du Canigou (les éditions 2000 à 2005), le Défi girondin (années 1990), le Grand Prix de France de descente de canyon en 1992, le Championnat de France de spéléologie en 1992, la Coupe Icare consacrée au vol libre (éditions 2006 à 2011).

\section{Définir et classifier en matière d'événementiel sportif}

10 Définir l'événementiel sportif n'est pas évident. Sans retenir la différenciation entre événement et manifestation que posent Bovy, Potier et Liaudat on peut repérer certains éléments notables dans la littérature. En management du sport, Ferrand (1993, p. 280), dans l'ouvrage fondateur de Loret (1993), estime que la généralisation du concept d'événement a provoqué sa banalisation et, partant, un affaiblissement de son sens. Il s'agirait selon lui "d'un fait social puissamment médiatisé, dont l'impact auprès des différents publics est lié à l'incertitude du résultat de l'action des différents acteurs placés dans une situation d'exploit, de performance ou de compétition. C'est pourquoi le sommet de l'événementiel est occupé par le trio Jeux olympiques, Coupe du monde de football et Championnat du monde de formule 1". Cette définition paraît juste, mais très restrictive. Elle exclut les événements ou manifestations dont l'objectif est de communiquer à travers le rassemblement festif, l'intégration du plus grand nombre, ou encore la mise en valeur d'un territoire à une échelle qui peut être globale mais aussi très locale. C'est le cas des événements sportifs organisés par des associations et des villes petites ou moyennes. On ne peut donc pas parler d'événement en soi, mais d'événement pour l'institution ou la communauté qui le produit (Desbordes et Falgoux, 2007). Didry (2008, pp. 18-20) - tout en ayant affirmé la difficulté à cerner la notion d'événement sportif - dégage un certain nombre de "caractéristiques transversales" autour de l'idée d'un rassemblement humain dans un lieu et à un moment particulier qui revêt un caractère exceptionnel par rapport au quotidien. Downward, Dawson et Dejonghe (2009) délimitent aussi un certain nombre de "variables significatives" pour appréhender un événement sportif : la fréquence (événement régulier ou irrégulier), le niveau de compétition, la nature (unisport ou multisport), le poids économique, le statut du porteur de l'événement (CIO, fédération sportive, entreprise commerciale, association) et sa localisation (fixe ou variable). Plus généralement, Weed (2008), Shipway et Fyall (2012), tout comme Didry (2008) et Ferrand (1993), s'accordent à dire qu'il s'agit d'un moment particulier d'émotion et de communion très fédérateur. Ce à quoi, Bolz (2014) ajoute, à partir d'une préoccupation historiographique, la présence d'enjeux identitaires importants. L'incertitude du résultat, chère au monde sportif selon Parlebas (1999), n'est par contre plus forcément au rendez-vous avec les spectacles de type show, démonstration ou exhibition, une forme de manifestation sportive ancienne mais qui perdure et se renouvelle (les indoors de windsurf de Bercy dans les années 1990, la Coupe Icare à Grenoble, ou encore différents événements de glisse urbaine à Strasbourg 
ou Bordeaux ces dernières années). Dans le monde marchand, selon les objectifs des concepteurs et la place qu'ils occupent dans la société, les événementiels organisés et les attentes en matière de communication seront différents.

11 Si on ne peut pas retenir toutes les facettes du concept d'événement en sciences sociales ${ }^{1}$ pour alimenter celui d'événement sportif - et notamment celui d'imprévisibilité mis en avant par Winock (2002) -, il est un aspect emprunté à l'histoire et à l'anthropologie qui n'est pas inintéressant pour un organisateur de compétitions sportives, celui de sa dimension mémorielle : l'idée qu'il en reste une trace matérielle, et surtout immatérielle. Comme le résume Farge (2002, p. 67), "'lévénement a une durée qui va bien au-delà de la simple temporalité des faits qui le constituent. Quand arrive un événement, il a été chargé par des perceptions et des sensibilités qui se sont formées avant qu'il ne survienne [...] de plus, il génère une mémoire. L'événement ne peut se définir qu'à l'intérieur d'un système complexe de temporalités". Autrement dit, pour qu'il y ait événement, il faut générer une certaine mémoire, ne serait-ce qu'au plan strictement sportif et à une échelle locale. Sans pour autant poser une définition quasiment impossible à formuler, nous envisageons un usage plus extensif du concept d'événement sportif tout en conservant une certaine ligne et les bénéfices de ces aspects conceptuels : une organisation préalable (par opposition à une situation accidentelle ou improvisée), une certaine limite de temps et de lieu (les courses autour du monde à la voile sont à la frontière d'un événement), une médiatisation ou du moins une certaine communication (par opposition à une réunion privée), une performance sportive (mais pas forcément compétitive) et des retombées (aussi bien matérielles, mémorielles que symboliques).

Face à cette effervescence événementielle, et à la suite notamment de Hall (1992), Getz (2007, 2013), Parent et Chappelet (2015), notre premier travail de chercheur a consisté à formuler une catégorisation des différentes formes d'événements sportifs existants ( $c f$. tableau 1). Cette catégorisation permet de différencier quelques grandes formes de projets, notamment: les compétitions strictement sportives organisées par des fédérations, des ligues professionnelles ou des organes du mouvement olympique; les spectacles de type show dans lesquels le résultat est prédéfini à l'instar d'une pièce de théâtre; les manifestations sportives de masse très libres et plus ou moins festives (on pense aux nombreuses courses sur route à pied, en vélo ou même en roller organisées par une ville ou par un département); les manifestations sportives assez libres mais fortement élitaires (physiquement, techniquement ou économiquement, comme les transats à la voile ou l'UTMB) ; et celles qui parviennent à combiner les deux aspects avec de simples participants en grand nombre auxquels s'ajoutent quelques performeurs qui viennent battre un record (les grands marathons des villes telles que Paris, Chicago, New York, Tokyo relèvent le plus souvent de cette catégorie; dans une certaine mesure, le Paris-Dakar aussi). 
Tableau 1. Essai de catégorisation des formes d'événements sportifs

\begin{tabular}{|c|c|c|c|c|}
\hline $\begin{array}{l}\text { Les compétitions } \\
\text { sportives }\end{array}$ & $\begin{array}{l}\text { Les spectacles } \\
\text { de type show }\end{array}$ & \multicolumn{3}{|c|}{$\begin{array}{l}\text { Les manifestations sportives } \\
\text { de masse.... et d'élite }\end{array}$} \\
\hline $\begin{array}{l}\text { de national à } \\
\text { international }\end{array}$ & de local à international & \multicolumn{3}{|c|}{ de local à international } \\
\hline $\begin{array}{c}\text { institutionnelle, } \\
\text { fédérale, olympique ou } \\
\text { marchande }\end{array}$ & marchande & \multicolumn{3}{|c|}{ associative, territoriale ou marchande } \\
\hline création ancienne & création ancienne & \multicolumn{3}{|c|}{ création récente } \\
\hline $\begin{array}{l}\text { calendrier national et } \\
\text { international contraint }\end{array}$ & ponctuel & \multicolumn{3}{|c|}{ absence de calendrier ou calendrier parallèle } \\
\hline motricité très codifiée & motricité plus libre & \multicolumn{3}{|c|}{ motricité libre à performative } \\
\hline $\begin{array}{l}\text { Les Jeux olympiques; la } \\
\text { Coupe du monde de } \\
\text { Football; le Tour de } \\
\text { France ; Roland-Garros, } \\
\text { les championnats } \\
\text { nationaux de chaque } \\
\text { discipline... }\end{array}$ & $\begin{array}{l}\text { Les matchs de catch ; } \\
\text { les tournées des } \\
\text { Harlem Globetrotters; } \\
\text { les indoors windsurf de } \\
\text { Bercy dans les } \\
\text { années } 1990 \text {; la Coupe } \\
\text { Icare à Grenoble ; } \\
\text { différents shows } \\
\text { urbains... }\end{array}$ & $\begin{array}{l}\text { Le Marathon du } \\
\text { Médoc; le } \\
\text { Marathon des } \\
\text { dunes; divers } \\
\text { événements } \\
\text { locaux... }\end{array}$ & $\begin{array}{l}\text { Le Paris-Dakar ; } \\
\text { les Rando Raid } \\
\text { SFR ; le Grand } \\
\text { Raid de La } \\
\text { Réunion... }\end{array}$ & $\begin{array}{c}\text { L'UTMB ; } \\
\text { le Raid } \\
\text { Gauloises; } \\
\text { le Marathon } \\
\text { des sables; les } \\
\text { défis } \\
\text { extrêmes... }\end{array}$ \\
\hline
\end{tabular}

13 Cette catégorisation a plusieurs fois évolué depuis nos premières tentatives à ce sujet (Gresser et Bessy, 1999; Bessy, 2005b ; Suchet, 2012), mais pour autant aucun de ces cadrages n'est vraiment satisfaisant dans la mesure où l'événementiel sportif est à la fois pluriel, en mutation constante, et surtout implique de multiples acteurs sur des territoires contrastés, en concurrence les uns avec les autres et tentant de se démarquer en innovant sans cesse pour "ne pas jouer dans la catégorie du concurrent". Autrement dit, les catégories sont en elles-mêmes un enjeu du secteur et se démultiplient au fur et à mesure des innovations événementielles participatives, écologiques, urbaines ou encore citoyennes : c'est le principe des champs (Bourdieu, 1992 ; puis Clivaz, Nahrath et Stock, 2011 ; Suchet, 2013). De nombreux événements illustrent cette démarche observable au niveau des innovations produites en matière de projet, de gouvernance et de communication. Notre production récente (Bessy, 2014a, 2014b) montre les bénéfices, mais aussi les limites de ce management de l'innovation pour les territoires.

\section{Les trois niveaux d'effet possible d'un événement sportif}

14 À la suite de différentes études en géographie, sociologie ou ethnographie du sport (Augustin, 2008a, 2009 ; Gumuchian, 1993 ; Barget et Gouguet, 2010 ; Chappelet, 2004, 2006 ; Fayos-Solá, 1997 ; Clivaz et Marcelpoil, 2009 ; Guibert, 2004 ; Bessy, 2014d; Tagsold, 2008 ; Bromberger, Hayot et Mariottini, 1995), et de la littérature relative aux festivals et à l'organisation de rassemblements culturels ou professionnels qui offre un point de vue intéressant à ce sujet (Gravari-Barbas et Veschambre, 2005 ; Crozat et Fournier, 2005 ; Di Méo, 2001 ; Christofle, 2013, 2004-2005), il s'agit d'étudier comment un événement temporaire peut s'ancrer dans le temps et dans l'espace pour fabriquer du territoire. Il en ressort différentes stratégies de développement. Indépendamment des matérialités de l'organisation, de l'équipement des sites ou des retombées financières, les auteurs en géographie sociale, notamment Gumuchian (1993), considèrent l'organisation des Jeux olympiques d'été ou d'hiver comme "une formidable machine à produire des images et 
construire des représentations du territoire". Il en est de même de toutes les grandes compétitions. Selon ces auteurs, la réussite d'un grand événement sportif se mesure à sa capacité d'avoir structuré une représentation cohérente à partir des multiples images produites, c'est-à-dire donner une cohérence à la profusion d'informations. On peut dès lors théoriquement considérer différents niveaux d'effets, à la fois au plan interne de leur emprise (dans la ville ou la région concernée) et au plan externe de leur rayonnement (hors de cette ville ou de cette région).

15 À un premier niveau, Durand (2006, p. 69) montre comment un événementiel sportif génère une fréquentation sur le terrain en légitimant le site parmi les pratiquants de l'activité, en lui faisant acquérir "une réputation, une notoriété". Cela reste néanmoins limité au registre sportif pour la ou les disciplines concernées et uniquement au site sportif considéré (la commune ou la région restant même parfois complètement méconnues). Il s'agit non seulement de faire connaître le site aux pratiquants, mais aussi de lui donner une valeur - qui est en quelque sorte proportionnelle au niveau de performance réalisée sur le site ou à la notoriété des compétiteurs qui s'y sont affrontés. Par exemple, l'aire de décollage de Saint-Hilaire-du-Touvet, à proximité de Grenoble, est de plus en plus connue à travers la Coupe Icare. Les parapentistes ou les deltistes étrangers viendront sur ce site dont ils ont entendu parler au travers de l'événement, de préférence à un autre. Les pratiquants d'escalade accorderont une grande importance à une voie comme Ali Baba, sur le site d'Aiglun, depuis son ascension médiatisée par Nina $\mathrm{Caprez}^{2}$; et on peut en dire de même des zones de plage du Mondial du vent à Leucate, dans le milieu du kitesurf; du gazon de l'Albatros chez les golfeurs; ou encore des lignes de descente en VTT de Hafjell pour les passionnés de downhill, depuis les championnats du monde à Lillehammer. Ce rayonnement peut être local ou mondial, mais il est restreint aux personnes qui pratiquent l'activité sportive concernée et, de fait, les retombées économiques sont souvent limitées. Plus encore, lorsqu'il s'agit d'une activité de niche et d'une compétition portée initialement par un petit groupe d'acteurs, la nouvelle fréquentation du site soulève parfois des conflits d'usage (de nombreux cas existent en escalade, en sports d'eau vive ou en VTT).

16 À un second niveau, un événementiel peut permettre à une zone - plus ou moins constituée en destination touristique - de se maintenir comme la destination de référence pour le secteur concerné (les sports d'hiver, les sports de nature, le tourisme littoral...). Une Coupe du monde de ski, des compétitions de patinage artistique ou une course de chiens de traîneau font partie de ces événements qui ne visent pas seulement le public pratiquant l'activité (le ski, le patinage ou le mushing), mais qui dynamisent le secteur des sports d'hiver sur l'ensemble de la zone concernée. Les retombées du tourisme sont plus importantes et plus diversifiées que sur une activité sportive précise et contribuent le plus souvent fortement à l'économie globale du lieu.

Enfin, à un troisième niveau, l'événementiel contribue à l'imagerie du lieu pour la société dans son ensemble. Au même titre que l'industrie, l'urbanisme, le paysage ou encore la recherche scientifique, l'organisation d'un rassemblement sportif peut alimenter la représentation socialement partagée du territoire et sa dynamique sociale au plan interne. Selon les disciplines sportives concernées, l'organisation d'une compétition majeure peut restituer l'idée d'un territoire mondain (golf, voile, cricket...), celle de l'intégration "black blanc beur" (football, basket...), la réussite entrepreneuriale et urbaine (formule 1, moto de circuit...), ou encore l'idée d'un territoire très jeune (surf, skateboard...). L'événement contribue dès lors à l'élaboration d'une image qui dépasse 
largement le cadre sportif ou touristique. Encore une fois, ce rayonnement peut être assez local ou mondial, mais il touche uniformément tout un chacun, intéressé ou pas par le sport. Chamonix-Mont-Blanc est un cas emblématique de bourgade inséparable de l'alpinisme et des activités de montagne - ce que l'invention des Jeux olympiques d'hiver, en 1924, a souligné et que, aujourd'hui, l'UTMB contribue à actualiser. À partir des écrits de Frémont (1987), Gumuchian (1993, p. 62) montre comment les Jeux olympiques de Grenoble, en 1968, s'inscrivent directement dans la constitution d'une représentation patiemment construite tout au long de la seconde moitié $d u x^{e}$ siècle pour faire de Grenoble une ville de l'innovation, de la jeunesse au travail et de la haute performance technologique française. Encore plus largement, l'étude coordonnée par Dauncey et Hare (1999) estime que la Coupe du monde de football 1998 en France a été une étape importante dans l'histoire du pays, pour son identité nationale et son rayonnement international. Autant dire que le lieu concerné peut varier d'un simple village à toute une nation, mais qu'il est illusoire de tenter une évaluation des retombées économiques dans la mesure où il s'agit d'une contribution inquantifiable à la vie sociale.

Si les deux dimensions contribuent indissociablement à la résonnance globale d'un événement sportif, niveau d'effet et niveau d'échelle ne doivent pas être confondus ( $c f$. tableau 2). Un événement ne dépassant pas l'échelle du village ou de la région comme les Corridas de Céret, au sud-ouest de Perpignan, bénéficie à la société locale, tandis qu'un événement tel que le Red Bull Rampage, organisé sur des reliefs semi-désertiques en Utah, est mondialement suivi des spécialistes de VTT mais ne bénéficie d'aucun effet en dehors de ces passionnés de descente extrême.

Tableau 2. Niveaux d'effet et niveau d'échelle dans la résonnance d'un événement sportif

\begin{tabular}{|c|c|c|c|c|}
\hline & Niveau c & échelle (dimension & e cette emprise et & e ce rayonnement) \\
\hline 范 & & Local & & Mondial \\
\hline 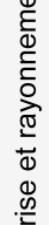 & $\begin{array}{r}\text { Limité à } \\
\text { l'activité ou à la } \\
\text { discipline } \\
\text { sportive }\end{array}$ & $\begin{array}{l}\text { La Course des Volcans } \\
2008 \text {, Clermont-Ferrand. } \\
\text { Sancy Verte } 2016 \\
\text { [épreuve de VTT], La } \\
\text { Bourboule. } \\
\text { Et autres compétitions } \\
\text { sportives très locales... }\end{array}$ & $\begin{array}{l}\text { Championnats } \\
\text { d'Europe de VTT } \\
\text { 2006, Limosano } \\
\text { (Italie). }\end{array}$ & $\begin{array}{l}\text { Le Petzl RocTrip 2011, Vallée du } \\
\text { Gêtu (Chine). } \\
\text { Le Red Bull Rampage 2015, } \\
\text { Virgin (Utah, USA). }\end{array}$ \\
\hline 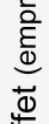 & $\begin{array}{l}\text { Sectoriel et } \\
\text { touristique }\end{array}$ & $\begin{array}{l}\text { Compétitions/animations } \\
\text { en stations de sport } \\
\text { d'hiver. }\end{array}$ & $\begin{array}{l}\text { La Grande Odyssée } \\
\text { Savoie-Mont-Blanc } \\
\text { 2010, Savoie et Haute- } \\
\text { Savoie. }\end{array}$ & $\begin{array}{l}\text { Le Mondial du Vent } 2010 \text {, } \\
\text { Leucate. } \\
\text { Le Lacanau Pro } 2014 \text { [surf], } \\
\text { Lacanau. }\end{array}$ \\
\hline 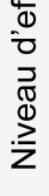 & $\begin{array}{r}\text { Étendu à } \\
\text { l'ensemble } \\
\text { de la société }\end{array}$ & $\begin{array}{l}\text { Céret de toros } 2008 \text { [Feria } \\
\text { et corridas], Céret. } \\
\text { Certaines petites } \\
\text { compétitions de pratiques } \\
\text { sportives pourtant } \\
\text { localement identitaires }\end{array}$ & $\begin{array}{l}\text { Le Grand Prix de la } \\
\text { Saint-Louis } 2010 \\
\text { [tournoi de joutes } \\
\text { languedociennes], } \\
\text { Sète. }\end{array}$ & $\begin{array}{l}\text { Coupe du Monde de football } \\
\text { 1998, Paris. } \\
\text { Les Jeux Olympiques d'été 2008, } \\
\text { Pékin (Chine). } \\
\text { Les compétitions sportives } \\
\text { mondiales majeures... }\end{array}$ \\
\hline
\end{tabular}

\section{Deux grandes stratégies d'orientation pour une organisation}

Presque indépendamment de ces trois niveaux d'effet, on peut mettre en évidence deux grandes stratégies d'orientation pour une organisation porteuse d'un événement sportif. Effectivement, lors du montage d'un événementiel sportif et touristique, les acteurs doivent s'entendre sur les modalités de participation aux épreuves: faut-il ouvrir les compétitions à un grand nombre d'équipes (inscriptions payantes, repas tarifés) ou bien 
conserver, pour chaque pays, les compétiteurs sélectionnés par les championnats nationaux et/ou les "têtes d'affiche" (sur le principe d'une invitation) ? Sur ce point, une étude minutieuse des arguments montre que derrière l'apparence d'une simple question, le débat concerne les stratégies d'utilisation des compétitions sportives en tant que moyens de développement territorial. D'un côté, les arguments d'une compétition ouverte à un grand nombre d'équipes tournent autour d'une conception économique directe et immédiate (le prix des inscriptions, les dépenses des compétiteurs et de leur famille) - c'est le modèle A, avec notamment tous les cas de rassemblements sportifs participatifs -, tandis que les défenseurs d'un strict élitisme sportif envisagent de développer une attractivité à long terme du territoire grâce à la construction d'une image et d'une symbolique particulière ou bien s'attachent uniquement aux questions sportives sans se préoccuper d'autre chose - c'est le modèle B, celui notamment de l'organisation des Jeux olympiques d'hiver ou d'été; mais aussi d'un championnat régional d'haltérophilie (cf. figure 1).

À la suite de Gresser et Bessy (1999) et de Didry (2008), quasiment seuls auteurs à mentionner cet aspect au sein d'une littérature pourtant abondante (exclusion faite des approches strictement économiques), on peut formaliser cette opposition entre modèles A et B. En fait, d'autres modèles, et notamment des solutions intermédiaires, existent. En sport, on pense au cas des marathons qui permettent autant de jouer sur le nombre de participants que sur une élite définie par l'ordre d'arrivée (Bessy, 1994, 2000 ; Lapeyronie, 2007). D'autres fois, le capital image injecté localement n'est pas fonction des performances réalisées ni d'une stricte sélection des participants, mais de la dimension novatrice, géopolitique ou particulièrement singulière du moment. Pour autant, on peut estimer que les deux grands modèles d'orientation formalisés ci-dessous ( $c f$. figure 1 ) structurent souvent les débats au moment du lancement d'un événement sportif, touristique, mais aussi scientifique ou artistique.

Figure 1. Formalisation par modèles d'orientation possibles d'un événementiel sportif

\begin{tabular}{|c|c|}
\hline Modèle A & Modèle B \\
\hline $\begin{array}{l}\text { Logique participative. Compétition ouverte } \\
\text { à un grand nombre d'équipes ou d'individuels } \\
\text { Inscriptions payantes, déplacement ou } \\
\text { hébergement au frais des participants. Appel à } \\
\text { participation. }\end{array}$ & $\begin{array}{r}\text { Élitisme sportif, pré-sélection, test } \\
\text { (ou participation sur invitation, "têtes } \\
\text { d'affiche"). } \\
\text { compétiteurs "tous frais payés", } \\
\text { voire rémunérés au cachet. }\end{array}$ \\
\hline $\begin{array}{l}\text { Peu ou pas de spectateurs et d'auditeurs } \\
\text { initialement (et souvent avec entrée libre) }\end{array}$ & $\begin{array}{l}\text { Spectateurs et auditeurs plus nombreux, } \\
\text { sur billets ou tickets d'entrée payants. }\end{array}$ \\
\hline Conception économique directe et immédiate. & $\begin{array}{l}\text { Participation à la construction d'une image } \\
\text { ou d'une symbolique. } \\
\text { Développement d'une attractivité à long } \\
\text { terme du territoire. }\end{array}$ \\
\hline $\begin{array}{l}\text { Retombées attendues : prix des inscriptions, } \\
\text { retombées induites par la venue des } \\
\text { compétiteurs et souvent de leur famille. }\end{array}$ & $\begin{array}{l}\text { Retombées attendues : mise en réseau } \\
\text { professionnel du territoire dans un secteur } \\
\text { donné, retombées très spécifiques, image, }\end{array}$ \\
\hline $\begin{array}{l}\text { Faibles dépenses (proportionnellement à la } \\
\text { taille de la manifestation), et retombées } \\
\text { prévues assurées mais souvent modestes. } \\
\text { Risques de déficits ou d'imprévus majeurs } \\
\text { faibles. }\end{array}$ & $\begin{array}{r}\text { Dépenses (parfois très importantes) sans } \\
\text { retombées assurées ni forcément } \\
\text { calculables. }\end{array}$ \\
\hline
\end{tabular}


21 À partir de ce schéma, on peut venir positionner plus ou moins précisément sur une droite des événements participatifs - telle l'Ardéchoise, en cyclisme, qui attire presque 16000 coureurs (droits d'inscription: environ 80 euros, sans compter les frais d'hébergement et de transport) devant un public seulement local ou apparenté aux participants qui assiste (librement) aux phases de la course sur le bord de la route - et des événements élitaires - tel le tournoi de Roland-Garros où s'affrontent deux joueurs sur le court (qui touchent un cachet d'environ un million d'euros chacun) devant un public de 15000 spectateurs (qui payent environ 200 euros leur place en tribune) ${ }^{3}$. Pour l'organisateur, il s'agit notamment de savoir : qui sont les clients? celui qui fait du sport? ou bien celui qui regarde le sport? Entre ces deux extrémités se trouvent bon nombre d'événementiels en situation intermédiaire ( $c f$. figure 2). Un même événement peut d'ailleurs évoluer au cours des éditions, comme les Jeux paralympiques organisés en accord avec le CIO peu après les Jeux olympiques d'été ou d'hiver, qui sont passés d'une compétition à faible enjeu sportif, quasiment sans financements et "où les tribunes était vides" à un événement médiatisé, sponsorisé et quelque peu rémunérateur "où les tribunes sont pleines"4. Au-delà de ce schéma initial dans le projet d'organisation, le nombre de spectateurs dépend évidemment d'un grand nombre de facteurs (nature de l'activité, culture de référence des personnes, accessibilité des épreuves en elles-mêmes...).

Figure 2. Modèles d'orientation et continuum de positionnement des événements sportifs

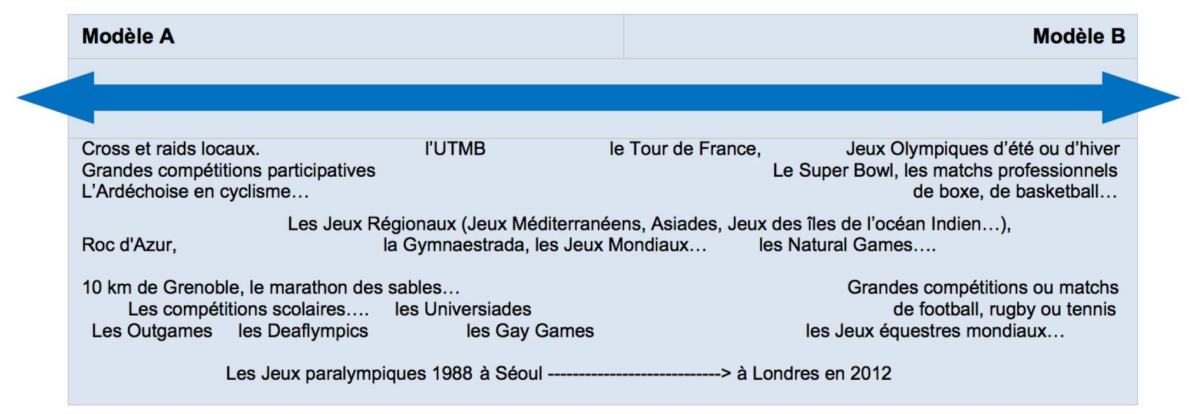

\section{Événements sportifs importés versus événements sportifs fabriqués}

Les Jeux olympiques, les championnats d'Europe ou les grands matchs de league aux USA, sans compter la Coupe du monde de football sont autant de grands événements qui tournent à travers le monde. Les villes tentent d'ailleurs d'attirer ces grands moments du sport médiatique contrôlé par des fédérations internationales, des leagues professionnelles, le CIO, ou même parfois un puissant sponsor. Depuis les années 1990, de nombreuses villes et pays ont même développé des stratégies spécifiques (plus ou moins transparentes et légales) et des politiques publiques d'accueil systématique de ces événements sportifs (Chappelet, 2006). Les villes rivalisent d'audace à partir de dossiers de candidature qui mobilisent parfois tout un pays. Ces manifestations d'un format prédéfini peuvent être qualifiées d'“événements importés". La ville et le pays héritent d'un modèle conçu en d'autres lieux par d'autres acteurs sur lequel ils n'ont pas ou peu d'emprise. Il en est de même au plan national avec les municipalités qui se disputent les 
étapes du Tour de France (une compétition historique française mais fortement standardisée, et qui n'a pas réellement d'itinéraire fixe : elle peut même s'exporter dans les pays voisins) : un investissement très coûteux pour de petites communes, mais censé produire un impact direct et indirect important ${ }^{5}$.

À l'opposé de ce format d'événements importés, on peut mettre en évidence l'existence d'événements nés localement et qui conservent une spécificité territoriale : ce que nous avons appelé des "événements fabriqués" (Bessy, 2014b, 2014a) et que Chappelet (2014) nomme des "événements sportifs patrimoniaux", dans le sens où ils font partie du patrimoine d'un lieu. ${ }^{6}$. Ces derniers ont un fort ancrage local, car ils ont été imaginés, développés, puis organisés chaque année par des acteurs locaux soucieux du dynamisme de leur territoire. Ils sont souvent plus accessibles aux masses, car davantage pensés sur la base d'engagements à géométrie variable (le Marathon du Médoc, l'Ardéchoise, Roc Azur, l'Open d'escalade Salève Annemasse...) ; quelques-uns sont aussi sur une attente sportive de très haut niveau (la très ancienne Coupe Spengler ou même Roland-Garros que Chappelet classe comme un événement patrimonial, mais aussi les Jeux pyrénéens de l'aventure au début des années 1990 et maintenant, progressivement, l'UTMB) ou bien affichent des droits d'entrée fortement élitistes (notamment en golf, en voile ou en équitation), ou encore se situent entre les deux (la très ancienne Patrouille des glaciers, les Natural Games, le Marathon de Paris ou ceux d'autres grandes villes du monde...). Dans un certain sens, le Tour de France, cité précédemment pour ses étapes, pourrait aussi être considéré au plan mondial comme un événement caractéristique du territoire français, historiquement et socialement signifiant (Boury, 1997; Mignot, 2014). En prenant de l'importance, de nouveaux acteurs économiques, politiques et sportifs entrent en jeu, mais toujours à partir d'un cadre initial spécifique au territoire et d'une gouvernance indissociable du lieu. Autant de caractéristiques qui permettent à ces événements fabriqués d'être - proportionnellement à leur taille - plus "impactants" pour les territoires concernés. Ces productions sportives - qui ne sont pas nouvelles mais se multiplient et se complexifient - sont à mettre en parallèle avec une diversification croissante de l'offre événementielle liée à un renouvellement du secteur qui déborde du champ sportif traditionnel pour atteindre le tourisme, la culture ou l'écologie. Plus encore, une nouvelle génération d'événements s'installe massivement dans le paysage en sortant de plus en plus de leurs espaces consacrés (stades, piscines, gymnases), à l'image du sport en lui-même caractérisé par une forte diversification territoriale (Augustin, 1987, 2002 ; Bessy et Hillairet, 2002 ; Loret et Waser, 2001). Cette génération d'événements se manifeste par un certain nombre d'innovations qui répondent à une demande toujours plus grande de loisirs et de tourisme sportifs, mais aussi aux nouveaux enjeux liés à l'intégration du sport dans une dynamique touristique de développement territorial (Bessy, 2014d). En effet, si les enjeux sportifs, économiques et politiques liés à la promotion d'une institution ou d'une région sont toujours présents, ils sont de plus en plus au service d'un enjeu global qui intègre aussi des dimensions socioculturelles, environnementales et territoriales. Cette attention renouvelée dans la manière de penser et de gérer les événements est liée à la recomposition et à la spécification des territoires, désireux de se construire une image dans le cas des nouvelles intercommunalités et de la décentralisation, et plus généralement dans un contexte de plus en plus concurrentiel (Joye et Leresche, 2006 ; Augustin, 2008b ; Clivaz, Nahrath et Stock, 2011 ; Suchet, 2013 ; Botti, 2011 ; Resmini et Torre, 2011). Ce tournant territorial survient à un moment où la mondialisation porte le risque d'uniformiser nos sociétés en alignant sur un même 
modèle, aux quatre coins de la planète, leurs mœurs et leurs mentalités, leurs manières de consommer et leur façon d'organiser l'espace et de l'habiter. Mais, bien que confrontées à ces forces qui les nivellent, les sociétés contemporaines n'abandonnent pas le parti de l'originalité. Plus que jamais, elles produisent de la différence, de la variété et de la singularité. L'événementiel en tant que temporalité d'une rencontre culturelle et sociale au sein des sociétés contemporaines en est un fragment et un symbole.

Figure 3. Degrés d'importation ou de fabrication d'un événement sportif

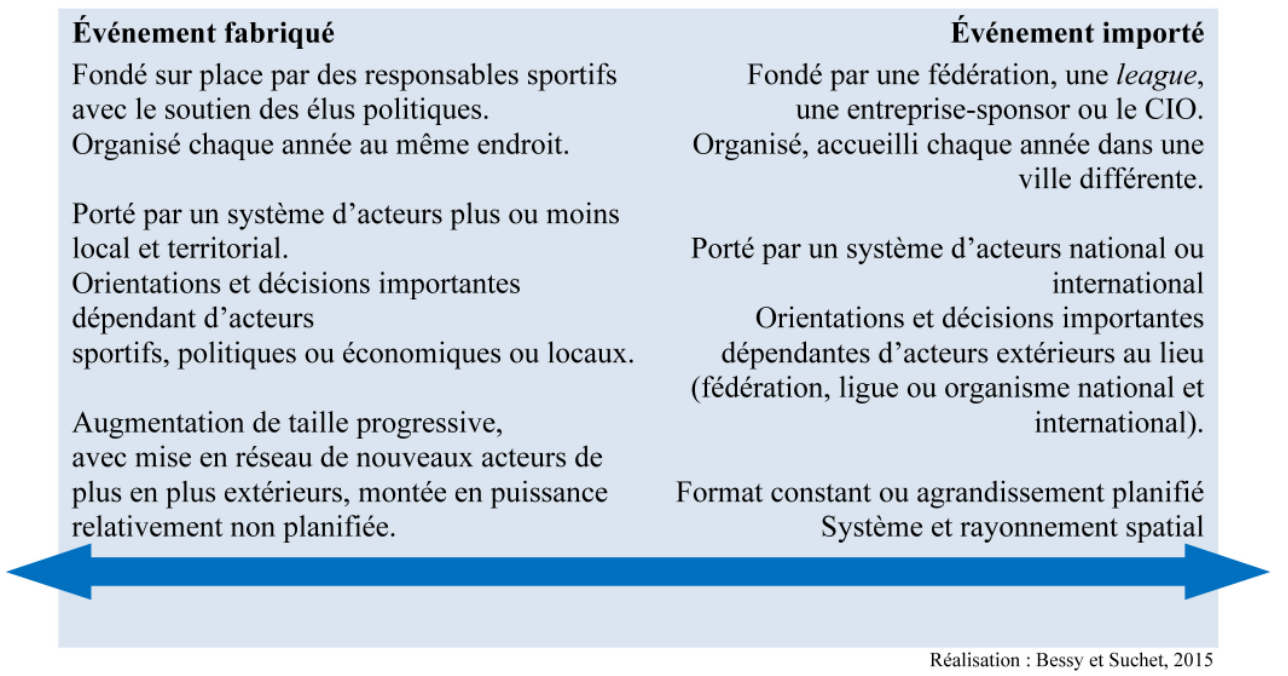

Mais, encore un fois, ce schéma d'opposition théorique se mue en continuum (cf. figure 3) dans la mesure où, en grandissant, certains événements imaginés localement deviennent de plus en plus contraints et parfois même commencent à s'exporter, tandis que des événements clairement importés tentent de parvenir à un certain ancrage local dans une intention de développement territorial des régions concernées ; sans compter qu'il existe des événements volontairement conçus dans une situation intermédiaire.

\section{Conclusion}

Approcher l'événementiel sportif nécessite un outillage conceptuel solide tant ce champ est aujourd'hui extrêmement diversifié, impliquant de nombreux acteurs aux intérêts à la fois convergents et divergents (fédérations, associations, entreprises, partenaires, collectivités...), revêtant de multiples formes (des plus institutionnalisées aux plus informelles, des plus sectorisées aux plus hybridées) et générant des enjeux plus ou moins importants sur les plans économiques, socioculturels, environnementaux, territoriaux, communicationnels et politiques. En l'occurrence, plutôt que de mobiliser ou de recommander un cadre déjà construit à partir d'autres objets, cette recherche a souhaité proposer un dispositif théorique, certes modeste, mais suffisamment robuste pour rendre compte de manière inédite de certains rapports entre un événement temporaire, des individus agissants et un espace territorialisé. Il s'agit de conceptualiser le niveau d'effet d'un événement, l'opposition structurelle entre événements importés et événements fabriqués, et enfin le choix d'une stratégie d'orientation plus ou moins participative. Si la première partie de ce travail pointe les difficultés à définir et à classifier les différents types d'événements sportifs, on espère des applications possibles de ces cadrages 
théoriques dans de futures études de cas. Il s'agira en quelque sorte de tester la part d'explication de ces trois modélisations d'une réalité sportive, touristique et culturelle nécessairement multiple. En souhaitant que ces futures études de cas puissent retirer de ce dispositif conceptuel quelques apports pratiques, voire aussi théoriques, ne serait-ce que pour en montrer les limites.

\section{BIBLIOGRAPHIE}

Jean-Pierre Augustin, "Le territoire dans tous ses états : extension des pratiques sportives et nouveaux espaces d'aventure", dans Sport et changement social, Maison des sciences de l'homme d'Aquitaine, 1987.

Jean-Pierre AUGUSTIN, "La diversification territoriale des activités sportives", L'Année sociologique , vol. 52, $\mathrm{n}^{\circ} 2,2002$.

Jean-Pierre AUGUSTIN, "Installations olympiques, régénération urbaine et tourisme", Téoros, vol. $27, n^{\circ} 2,2008$.

Jean-Pierre Augustin, "Le renouveau des territoires", dans Olivier BESSY (dir.), Sport, loisir, tourisme et développement durable des territoires, Presses universitaires du sport, 2008b.

Jean-Pierre Augustin, “Les Jeux olympiques, l'événement spatial par excellence”, Bulletin de l'Association de géographes français, vol. 86, n 3, 2009.

Éric BARGET et Jean-Jacques GOUGUET, Événements sportifs. Impacts économique et social, De Boeck, 2010.

Alban BENSA et Eric FASSIN, “Les sciences sociales face à l'événement”, Terrain, n 38, 2002.

Olivier BESSY, 10 ans de fête : Marathon des châteaux du Médoc et des Graves, Delteil, 1994.

Olivier BESSY, "Le Marathon du Médoc ou le Carnaval de la course à pied. Du sport apollinien au sport dionysiaque", dans Sport, fête et société, coll. "Les cahiers de l'université sportive d'été", Maison des sciences de l'homme d'Aquitaine, $\mathrm{n}^{\circ}$ 9, 1995.

Olivier BESSY, "Le Marathon du Médoc. Un événement sportif au cœur du développement local”, Cahier Espaces, $\mathrm{n}^{\circ}$ 6, 2000.

Olivier BESSY, Le Grand Raid de La Réunion. À chacun son extrême et un emblème pour tous, Océan éditions, 2002.

Olivier BESSY, "Sociologie des pratiquants de l'extrême : le cas de figure des participants au Grand Raid de La Réunion", STAPS, vol. 26, n 69, 2005a.

Olivier BESSY, Sports, loisirs et société. De la diffusion sociale à la construction identitaire. De l'innovation marketing au développement durable des territoires, mémoire du diplôme d'HDR, université de La Réunion, 2005b.

Olivier BESsy, The North Face, Ultra-Trail du Mont-Blanc : un mythe, un territoire, des hommes, Le Petit Montagnard / Autour du Mont-Blanc, 2012. 
Olivier BESSY, "De l'innovation événementielle à l'innovation territoriale", dans Olivier BESSY (dir.), L'Innovation dans l'événementiel sportif. De l'attractivité touristique au développement territorial, Presses universitaires du sport, 2014a.

Olivier BESSY, "Le management de l'innovation dans l'événementiel sportif", Revue européenne de management du sport, $\mathrm{n}^{\circ} 42,2014 \mathrm{~b}$.

Olivier BESSY, "The North Face, Ultra-Trail du Mont-Blanc : une ressource territoriale pour l'Espace Mont-Blanc et Chamonix", dans Olivier BESSY (dir.), L'Innovation dans l'événementiel sportif. De l'attractivité touristique au développement territorial, Presses universitaires du sport, 2014c.

Olivier BESSY (dir.), L'Innovation dans l'événementiel sportif. De l'attractivité touristique au développement territorial, Presses universitaires du sport, $2014 \mathrm{~d}$.

Olivier BESSY et Dieter HILlAIRET (dir.), Les Espaces sportifs innovants, Presses universitaires du sport, 2002, 2 vol.

Olivier BESSY et Olivier NARIA, "La provenance des participants au Grand Raid de La Réunion", M@ppemonde, $\mathrm{n}^{\circ}$ 74, 2004.

Daphné воLz, “L'événement en histoire culturelle du sport : essai d'historiographie", Science \& Motricité, vol. 86, $\mathrm{n}^{\circ}$ 4, 2014.

Laurent вотті, Pour une gestion de la touristicité des territoires, Balzac éditeur, 2011.

Pierre Bourdieu, “La logique des champs", dans Pierre Bourdieu et Loïc J. D. WACQUANT (dir.), Réponses, Seuil, 1992.

Paul Boury, La France du Tour : le Tour de France, un espace sportif à géographie variable, L'Harmattan, 1997.

Philippe воvy, Françoise POTIER et Chirsitan LIAUDAT, Les grandes manifestations : planification, gestion des mobilités et impacts. L'Aube, 2003.

Christian BROMBERGER, Alain HAYOT et Jean-Marc MARIOTTINI, Le Match de football : Ethnologie d'une passion partisane à Marseilles, Naples et Turin, Maison des sciences de l'homme, 1995.

Jean-Loup CHAPPELET, "Événements sportifs et développement territorial”, Revue européenne de management du sport, $\mathrm{n}^{\circ} 12,2004$.

Jean-Loup CHAPPELET (dir.), Les Politiques publiques d'accueil d'événements sportifs, L'Harmattan, 2006.

Jean-Loup CHAPPELET, "Les événements sportifs patrimoniaux comme vecteur de développement territorial", dans Olivier BESSY (dir.), L'Innovation dans l'événementiel sportif. De l'attractivité touristique au développement territorial, Presses universitaires du sport, 2014.

Sylvie CHRISTOFLE, "Stratégies métropolitaines et tourisme de réunion et de congrès international en France", Territoires en mouvement (anciennement Hommes et Terres $d u$ Nord), nº 2 , 2004-2005.

Sylvie CHRISTOFLE, "Tourisme de réunions et de congrès et recherche géographique. Une thématique en devenir", Mondes du tourisme, hors-série, mars 2013.

Christophe CLIVAz et Emmanuelle MARCELPOIL, "Big Sport Events and Governance. The Case of Alpine World Ski Championships in three Destinations", dans Valentina CASTELLANI et Serenella SALA (dir.), Sustainable Tourism as a Factor of Local Development, Tangram, 2009. 
Christophe clivaz, Stéphane NaHRath et Mathis stock, "Le développement des stations touristiques dans le champ touristique mondial", Mondes du tourisme, hors-série, septembre 2011.

Dominique crozAT et Sébastien FOURNIER, "De la fête aux loisirs : événement, marchandisation et invention des lieux", Annales de Géographie, vol. 114, n 643, 2005.

Hugh DAUNCEY et Geoffrey Edward HARE (dir.), France and the 1998 World Cup. The National Impact of a World Sporting Event, Frank Cass, 1999.

Michel DESBORDES, "A review of the economic impact studies done on the Tour de France: methodological aspects and first results", International Journal of Sport Management and Marketing, vol. 2, n 5-6, 2007.

Michel DESBORDES et Julien FALGoux, Organiser un événement sportif, Éditions d'Organisation, 2007.

Guy DI MÉo (dir.), La Géographie en fêtes, Ophrys, 2001.

Nico DIDRY, Les Enjeux de l'événement sportif, L'Harmattan, 2008.

Paul DownWARD, Alistair Dawson et Trudo Dejonghe, Sports Economics: Theory, Evidence and Policy, Elsevier, 2009.

Dominique DURAND, "L'événementiel en parapente au service du développement économique", dans Olivier BESSY (dir.), Loisirs sportifs de nature, nouvelles technologies et développement touristique durable à La Réunion, actes de colloque, université de La Réunion, 2006.

Arlette FARGE, "Penser et définir l'événement en histoire. Approche des situations et des acteurs sociaux", Terrain, $\mathrm{n}^{\circ} 38,2002$.

Eduardo FAYos-solá, “The impact of mega events”, Annals of Tourism Research, vol. 25, n 1, 1997.

Alain FERRAND, "La communication par l'événementiel sportif : entre émotion et rationalité", dans Alain LORET (dir.), Sport et management. De l'éthique à la pratique, Dunod, 1993.

Armand FRÉMONT, "Milieu géographique et innovation : le cas grenoblois", Revue de géographie alpine, vol. 75, $\mathrm{n}^{\circ} 4,1987$.

Donald GETZ, Event Studies: Theory, Research and Policy for Planned Events, Butterworth-Heinemann, 2007.

Donald GETZ, Event Tourism: Concepts, International Case Studies, and Research. Cognizant Communication, 2013.

Maria GRAVARI-BARBAS et Vincent VESCHAMBRE, "S'inscrire dans le temps et s'approprier l'espace : enjeux de pérennisation d'un événement éphémère. Le cas du festival de la BD à Angoulême", Annales de Géographie, vol. 114, nº 643, 2005.

Bernard GRESSER et Olivier BESSY, "Le management d'un événement sportif", dans Gisèle LACROIX et Anne-Marie WASER (dir.), Le Management du sport. 15 études de cas corrigées, Éditions d'Organisation, 1999.

Christophe GUIBERT, "La Coupe de l'America ne viendra pas à Marseille", Revue Espaces, n 217, 2004.

Colin Michael HALL, Hallmark Tourist Events: Impacts, Management and Planning, Belhaven Press, 1992.

Hervé GUMUCHIAN, “Des représentations spatiales aux retombées économiques : les Jeux olympiques d'hiver", dans Bernard DEBARBIEUX et Hervé GUMUCHIAN (dir.), Géographie économique et représentations, Anthropos, 1993. 
Dominique JoYe et Jean-Philippe LERESCHE, "Le retour des territoires", dans Patrick LE GALÈS (dir.), Gouvernement et gouvernance des territoires, La Documentation française, 2006.

Leo JAGo et Robin N. SHAW, "Special events: A conceptual and differential framework", Festival Management \& Event Tourism, vol. 5, nº 1-2.

Johanne KAY et Suzanne LABERGE, "The 'New' Corporate Habitus in Adventure Racing", International Review for the Sociology of Sport, vol. 37, $\mathrm{n}^{\circ}$ 1, 2002.

Gisèle LACRoIx, “Sport-aventure et lien social : l'exemple du Raid Passion Hérault", Corps et culture, $\mathrm{n}^{\circ} 3,1998$.

Bruno LAPEYRonie, Analyse socio-économique des processus de développement des pratiques sportives: l'exemple des marathons en France, thèse de doctorat, université Paris 11, 2007.

Alain LORET (dir.), Sport et management. De l'éthique à la pratique, Dunod, 1993.

Alain LORET et Anne-Marie WASER (dir.), Glisse urbaine, Autrement, 2001.

Jean-François MIGNOT, Histoire du Tour de France, La Découverte, 2014.

Olivier OBIN et Jean CORNELOUP, "Fabrique territoriale d'un événementiel en sport de nature : les 'Natural Games' de Millau”, dans Olivier BESSY (dir.), L'Innovation dans l'événementiel sportif. De l'attractivité touristique au développement territorial, Presses universitaires du sport, 2014.

Ignace olAZABAL et Joseph LÉvy (dir.), L'Événement en anthropologie. Concepts et terrains, Presses de l’Université Laval, 2006.

Milena M. PARENT et Jean-Loup CHAPPELET (dir.), Routledge Handbook of Sports Event Management, Routledge, 2015.

Pierre PARLEBAS, Jeux, sports et sociétés, Insep, 1999.

Mireille PRESTINI-CHRISTOPHE, "La notion d'événement dans différents champs disciplinaires", Pensée plurielle, vol. 13, n 3, 2006.

Laura RESMINI et André TORRE (dir.), Competitività territoriale: determinanti e politiche, Franco Angeli, 2011.

Richard SHIPWAY et Alan FYALL (dir.), International Sports Events: Impacts, Experiences and Identities, Routledge, 2012.

André sUCHET, Acteurs, gouvernance et dynamiques de projet dans la concurrence des territoires en tourisme. Étude autour du programme sportif "Pyrénées : laboratoire de l'olympisme" 1988-1994 et des Jeux pyrénéens de l'aventure 1993, thèse de doctorat, université de Grenoble, 2012.

André SUCHET, "Le concept de champ pour une approche interdisciplinaire du tourisme. Discussion théorique à propos de Clivaz, Nahrath et Stock (2011), et perspectives de généralisation", Mondes du tourisme, $\mathrm{n}^{\circ}$ 8, décembre 2013.

André SUCHET et Dominique JoRAND, "Le projet territorial des Jeux pyrénéens de l'aventure 1993 : retour sur l'ambition du programme ‘Pyrénées : laboratoire de l'olympisme”', dans Olivier BESSY (dir.), L'Innovation dans l'événementiel sportif. De l'attractivité touristique au développement territorial, Presses universitaires du sport, 2014.

André SUCHET, Dominique JORAND et John TUPPEN, “The history and geography of a forgotten olympic project: The Spring Games", Sport in History, vol. 30, nº 4, 2010.

Christian TAGSOLD, Spiel-Feld: Ethnografie der Fussball-Weltmeisterschaft 2006, UVK Verlag, 2008. 
Mike WEED, Olympic Tourism, Butterworth-Heinemann, 2008.

Michel winock, “Qu'est-ce qu'un événement ?”, L’Histoire, vol. 268, n 9, 2002.

\section{NOTES}

1. Pour une revue de la littérature à ce sujet, nous avons notamment consulté PrestiniChristophe (2006), Bensa et Fassin (2002), Olazabal et Lévy (2006).

2. Une situation comparable à un événementiel sportif sachant que les compétitions d'escalade ne se pratiquent plus en extérieur depuis la fin des années 1990.

3. Sources: pour les données sur les Internationaux de France de tennis dits (tournoi de) "Roland-Garros" http://rolandgarros.fft-tickets.com/infos-billetterie/offres-et-tarifs ; http:// www.linternaute.com/sport/tennis/roland-garros-combien-gagne.shtml (on considère la finale), Pour la course cycliste l'Ardéchoise : http://blog.ardechoise.com/engagement/inscription-enligne/engagement-2/

4. "Paralympiques : stades pleins, plus de 2,4 millions de billets vendus", Le Nouvel Observateur, 31 août 2012 (version en ligne). Interview de David Smetanine par Benoît Pavan: "La médiatisation des Jeux paralympiques sera exceptionnelle", Le Monde, 7 mars 2014 (version en ligne).

5. Pour une revue de la littérature à ce sujet, voir Desbordes (2007), et pour une source avec des chiffres récents, voir l'article : “Accueillir le Tour de France, l'investissement qui paie”, Le Figaro, 6 juillet 2013 (version en ligne).

6. La littérature anglo-saxonne ne dresse pas clairement cette différenciation. Tout du moins, le concept parfois considéré comme équivalent de hallmark events ne bénéficie pas d'une définition stabilisée entre les auteurs (voir notamment Jago et Shaw, 1998 ; Hall, 1992 ; Getz, 2007).

\section{RÉSUMÉS}

L'organisation d'une grande compétition sportive est considérée à juste titre comme un moyen possible de développement, mais encore faut-il s'entendre sur le type de développement auquel on veut parvenir et sur les moyens dont on se dote pour ce faire. Effectivement, si les liens entre sport, tourisme et développement ont fait l'objet de nombreuses études en sciences de gestion, il reste un certain nombre d'aspects à mieux théoriser au plan social et culturel. À partir des résultats de plusieurs études de cas menées en France sur la période 1990-2014, cet article montre les difficultés à définir et à classifier les différents types d'événements sportifs, puis formalise trois axes d'un dispositif théorique. Il s'agit de conceptualiser le niveau d'effet d'un événementiel, l'opposition structurelle entre événements importés et événements fabriqués, et enfin le choix d'une stratégie d'orientation plus ou moins participative.

The organization of a major sporting event is rightly seen as a possible way of development, but we have to see what kind of development and how? Indeed, the links between sport, tourism and development have been the subject of numerous studies in management sciences, but there are a number of aspects that need to be taken into account in order to better theorize the social and cultural level. Based on the results of several case studies over the period 1990-2014, this article 
shows the difficulties to define and classify the different types of sporting events and formalizes three axes of a theoretical device. This is to conceptualize the effect level of an event, the structural opposition between imported events and manufactured events, and finally the choice of a more or less participatory strategy.

INDEX

Keywords : sport event, tourism, territorial development, strategy, organisation

Mots-clés : événement sportif, tourisme, développement territorial, stratégie, organisation

\section{AUTEURS}

\section{OLIVIER BESSY}

Professeur des universités, laboratoire SET (UMR 5603), Université de Pau et des Pays de l'Adour/ CNRS/Institut de recherches sur les sociétés et l'aménagement, Pau.

\section{ANDRÉ SUCHET}

Post-doctorant (programme CMIRA), Grupo de investigación de análisis territorial y estudios turísticos (GRATET)/Département de géographie de la Universidad Rovira i Virgili, Tarragone (Espagne) 